\title{
A mathematical analysis of physiological and morphological aspects of wound closure
}

\author{
E. Javierre - F. J. Vermolen • C. Vuik • \\ S. van der Zwaag
}

Received: 26 February 2008 / Revised: 17 November 2008 / Published online: 20 December 2008 (C) The Author(s) 2008. This article is published with open access at Springerlink.com

\begin{abstract}
A computational algorithm to study the evolution of complex wound morphologies is developed based on a model of wound closure by cell mitosis and migration due to Adam [Math Comput Model 30(5-6):23-32, 1999]. A detailed analysis of the model provides estimated values for the incubation and healing times. Furthermore, a set of inequalities are defined which demarcate conditions of complete, partial and non-healing. Numerical results show a significant delay in the healing progress whenever diffusion of the epidermic growth factor responsible for cell mitosis is slower than cell migration. Results for general wound morphologies show that healing is always initiated at regions with high curvatures and that the evolution of the wound is very sensitive to physiological parameters.
\end{abstract}

Keywords Wound closure kinetics - EGF diffusion - Moving interface - Level set method

Mathematics Subject Classification (2000) $\quad 35 \mathrm{~K} 57 \cdot 65 \mathrm{C} 20 \cdot 92 \mathrm{C} 17$

\footnotetext{
E. Javierre $\cdot$ S. van der Zwaag

Fundamentals of Advanced Materials, Delft University of Technology, Kluyverweg 1, 2629 HS Delft, The Netherlands

e-mail: s.vanderzwaag@tudelft.nl

E. Javierre $(\bowtie)$

CIBER-BBN Centro de Investigación Biomédica en Red en Bioingeniería, Biomateriales y Nanomedicina, Zaragoza, Spain

e-mail: etelvina.javierre@unizar.es

F. J. Vermolen · C. Vuik

Delft Institute of Applied Mathematics, Delft University of Technology, Mekelweg 4, 2628 CD Delft, The Netherlands

e-mail: f.j.vermolen@tudelft.nl

C. Vuik

e-mail: c.vuik@tudelft.nl
} 


\section{Introduction}

Mechanical injury to an organ may be produced by trauma or be the result of surgery. In any case, its successful healing is crucial to maintain (or even recover) the organ functionality and integrity. Understanding the biological, chemical and mechanical processes involved in wound healing is necessary to apply personalized healing therapies that may on one hand speed up the healing process achieving maximal function recovery in shorter times, and on the other hand help in preventing secondary effects such as aesthetic scars (keloids or hypertrophic scars), emotional distresses and poor quality of life often suffered by patients with chronic wounds. To this aim, many authors have made an effort in describing the processes involved in wound healing $[20,22,26,32,40,41]$, among many others, and successful therapies [14,31]. In most of the cases these processes depend on wound location, patient age, patient physiology, etc.

Healing of cutaneous wounds can be divided into three partly overlapping phases: inflammation, tissue formation and tissue remodeling [13]. In the inflammatory phase, the blood clot is formed achieving wound homeostasis, providing a provisional extracellular matrix for cell migration and a rich pool of cytokines. Platelets shed onto the wound site as result of vascular disturbance secrete chemokines (PDGF, EGF, TGF- $\beta$ ) that recruit inflammatory cells, macrophages and fibroblasts [41]. Neutriphils and leukocytes (which are immune response cells) clean the wound of foreign debris and prevent infections. During the tissue formation phase, re-epithelialization, angiogenesis, fibroplasia and wound contraction occur. Epithelial cells on the border of the wound undergo phenotype transformation and become motile by dissolution of intracellular desmosomes and arrangement of cytoplasmic actin filaments. Cell mobilitation is due to the so-called free edge defect or loss of contact inhibition, and is stopped when two or more cells come in contact [41].

Two significantly different cell motion modes have been observed in wound healing: lamellipodia crawling and actin cable contraction. Lamellipodia crawling occurs when cells on the wound edge develop small finger-like microvilli rich of actin filaments and migrate by adhesion of these microvilli to the substrate followed of cell contraction in the direction of filopodia [23]. Lamellipodia crawling is observed in adult epidermal wound healing [26] and corneal wounds [10]. However, in embryos, cell motion and hence wound healing proceeds differently: an actin cable is rapidly formed at the wound margin, and the wound closes by contraction of this cable (due to circumferential tension) dragging the surrounding cells behind. Healing by actin cable contraction is completed within a few hours, leaves no scar and the wound margin is smooth during healing [22]. Moreover, there is evidence that both types of cell motion are self-exclusive to a high degree. Thus, Brock et al. [9] demonstrated that when the formation of the actin cable is disrupted in embryo chick wounds, cells develop lamellipodia and the healing process may be compromised. Alternatively, Grasso et al. [16] showed that the actin cable is not formed when the extracellular matrix is preserved after wounding in in vitro cultures of corneal endothelial cells, and lamellipodiainduced migration was obtained independently of wound shape or size. The migration mechanism may as well be influenced by the size of the wound-significantly smaller 
in embryos [9,26] — and growth factor levels — much lower in embryonic wounds [36], which allows for a stronger influence of mechanical constrains.

Independently on the migration mechanism, an increased cell proliferation is needed behind the actively migrating cells to support wound closure [40]. Activated macrophages release a large number of molecules with angiogenic activity (PDGF, VEGF and FGF, among many others), which stimulate the migration and mitosis of endothelial cells and vascular growth [5]. Oxygen and nutrients are involved in many of the healing processes, from oxidative killing of bacteria to ATP levels maintenance to support cell function and protein synthesis [31], making angiogenesis critical to healing. Platelets, macrophages and secreted growth factors induce fibroblasts ingrowth and proliferation a few days after injury. Subsequently, fibroblasts activity consists of synthesis and deposition of collagen, which polymerizes into collagen fibers that when covalently cross-linked increase the tensile strength of the tissue. Wound contraction is due to connective-tissue compaction when fibroblast acquire myofibroblast phenotype, stimulated by TGF- $\beta$ and PDGF. In humans, around a $20 \%$ of wound size reduction occurs due to wound contraction whereas in loose-skinned animals the contraction may reach up to $90 \%$ [27]. Finally, once collagen homeostasis is reached, collagen fibers are reorganized according to local mechanical factors. In this last phase, the tensile strength of the undamaged tissue recovers up to 80\% [41] and more acellular tissue is eventually obtained by apoptosis of superfluous cells.

Mathematical modelling combined with computer simulations can help understanding the healing process and enlighten optimal conditions for treatments Furthermore, models of healing mechanisms in nature are of inspiration in the development of materials with self-healing properties [43]. The monographs by Britton [7] and Murray [25] are a good introductions to mathematical modelling of biological processes, whereas Sherratt and Dallon [35] give an exhaustive review of the state-of-the-art mathematical models of some specific processes involved in wound healing. Sherratt and Murray [37] couple for the first time epidermal cell proliferation with the effect of an inhibitory or activator autocrine growth factor, and study the effect of exogenous application of the growth factor on the healing response in planar epidermal wounds. Along the same line, Wearing and Sherratt [45] model the effect of keratinocyte growth factor on the dermis-epidermis interactions during epidermal wound healing. Chaplain [12] models the growth of the vasculature towards a tumor by the chemotactic action of TAF and incorporating the role of the extracellular matrix on the cellular migration pattern through haptotaxis. The direction of preferential movement of endothelial cells is determined form the microenvironment conditions. Gaffney et al. [15] model angiogenesis distinguishing between sprouts and blood vessels. Sprouts are formed from blood vessels and grow towards the regions of low vascularity, whereas blood vessels do not have the ability to migrate and passively follow the sprouts. Dynamic merging of sprouts is also included in the model. Wound contraction by the action of fibroblast and myofibroblast is modeled by Olsen et al. [27], where the wound and surrounding tissue is treated as a linear and viscoelastic material, and traction forces exerted by cells onto the underlaying tissue are incorporated.

The models presented in above-mentioned references are specific for single processes of wound healing in adult mammals. However, the processes described above 
are intrinsically interconnected and influence each other during healing in a nonstraightforward manner. Coupling their effects in a single model for healing phenomena is not easy and it has taken a long time for models to be formulated. There is also a limited knowledge of the role of wound shape (and size) on the healing kinetics, since most of the analysis carried out so far is restricted to axisymmetric wound shapes (i.e. planar or circular wounds). To our knowledge, only Sherratt and Murray [38] made an attempt to tackle the change in the evolution of the wound shape during healing as a response of the mitotic function of the growth factors. They observed that when the growth factors inhibit cell mitosis, ovate wounds evolve towards more elongated shapes as flat parts of the wound heal faster due to the greater accumulation of the mitosis inhibitor at the convex ends. Models of wound healing in embryos are also scarcely developed. Sherratt et al. [36] model the formation of the actin cable after injury by balancing the traction forces exerted by the substratum with the net forces on the actin filaments. However, the wound evolution is not considered in their model.

In the present paper, we extend the work by Adam and co-workers. Adam [3] applied the principles of a tumour growth model [2] to tissue regeneration. The model was based exclusively on the distribution of a mitogenic growth factor (that here we identify with EGF). Contrarily to the models described above, Adam did not incorporate any cellular density. Adam [3] considered only planar wounds on planar surfaces. Subsequently, circular wounds on planar surfaces [6] and circular wounds on spherical surfaces - as an approach to the skull cap-[4] were studied. In all these references, only the conditions for healing initiation were considered, in order to perform predictions on the so-called critical size defect. The time evolution of the wound was always disregarded. In a following paper, Vermolen et al. [44] studied the growth factor distribution prior to closure on elliptical wounds, whereas the wound evolution during healing was only tackled for circular wounds under the assumption of diffusive equilibrium. In the present work, we incorporate the time dependence of the problem to capture the evolution of the wound during healing. Moreover, we consider the wound morphology as key parameter on the healing kinetics, and present a computational model based on well known level set method designed to deal with arbitrary wound shapes. In our opinion, substantial knowledge on the healing process may be gained by incorporating the wound shape in the analysis.

The paper is organized as follows. First, the chemical model of wound closure is presented in Sect. 2 for general wound geometries. Subsequently, the computational method is described in mathematical rigor in Sect. 3. A Narrow Band Level Set Method [1] is used to capture the wound margin in time. A standard Galerkin Finite Element Method, with adaptive local grid refinement around the wound edge, is implemented to compute the EGF concentration at the wound edge more accurately and efficiently. Afterwards, analytic solutions and conditions for complete healing are described for certain asymptotic cases in Sect. 4, revealing parameter groupings that decide the success of healing. Next, in Sect. 5 we demonstrate the large influence of the physiological and geometrical parameters of the model on the healing kinetics. Finally, the consequences of our study are presented in Sect. 6, and the full list of parameters, physiological and numerical, used in our calculations is given in the Appendix. 


\section{The mathematical model for wound closure}

Epidermal wound closure in adults is entirely due to cell mitosis and migration. The mitotic activity is mainly responsible for the thickening of the epidermis, once the continuity of the basal membrane has been re-established. Since the thickness of the epidermis is very small compared to the wound dimensions, we will assume here that the wound is two dimensional. Hence, we will focus on the closure (i.e. re-epithelialization) of the basal membrane by cell mitosis and lamellipodia-induced migration, and disregard the thickening step.

In order to simplify the model, we consider a generic epidermic growth factor (EGF) that stimulates the increase of cellular activity in a layer surrounding the wound edge. The domain of computation $\Omega$ consists of the wounded region $\Omega_{w}$, which is surrounded by the active layer $\Omega_{\mathrm{al}}$ and the undamaged tissue $\Omega_{u}$ in the outer part, i.e. $\bar{\Omega}=\cup_{i} \bar{\Omega}_{i}$ and $\Omega_{i} \cap \Omega_{j}=\emptyset$ for $i, j \in\{w, a l, u\}$ and $i \neq j$. The concentration $c$ of the EGF within $\Omega$ is determined by the following reaction-diffusion equation

$$
\frac{\partial c}{\partial t}-D \Delta c+\lambda c=P \chi_{\Omega_{\mathrm{al}}(t)}
$$

which states the diffusional transport of the EGF through the epidermal tissue, its depletion or natural decay and its production. The characteristic function $\chi$, which only has support in $\Omega_{\mathrm{al}}(t)$, is used to account for the production of the EGF since it only takes place inside the active layer (i.e. by the band of cells surrounding the wound, [40]). Hence, the model described here does not incorporate growth factor signals between the dermis and the epidermis, such as KGF. For a model that deals with this kind of communication we refer to [45]. The parameters $D, \lambda$ and $P$ respectively denote the diffusion, decay and production rates of the EGF. At the outer boundary of the domain $\Omega$ we impose a no-flux boundary condition, i.e. $\nabla c \cdot \mathbf{n}=0$ where $\mathbf{n}$ denotes the normal vector.

Wound closure occurs by migration of cells into the center of the wound. We define the wound edge $\Gamma$ as the advancing front of cells. We assume that cells become motile if the accumulated EGF concentration exceeds a threshold value $\theta$, as in classical models of tissue regeneration and tumour growth $[8,17,18,39]$. This discontinuous switch mechanism is just a limiting case for the coupling of EGF concentration and cell mitosis through a sigmoid function. Investigation of the effect of the slope in the sigmoid function on the healing behaviour may be interesting, but departs from the aim of the present work. In addition to this, cell migration may be interrupted if the EGF concentration drops below this threshold value. Therefore, in this approach cell motility is dose-dependent. Moreover, we assume that the closure rate is proportional to the local curvature of the wound edge [44], which is in line with the observations made by Buck [10]. Hence, the normal velocity $v_{n}$ of the wound edge $\Gamma$ is given by

$$
v_{n}=(\alpha+\beta \kappa) H(c-\theta)
$$

where $\alpha \geq 0$ and $\beta \geq 0$ (and $\alpha+\beta>0$ ) denote the coefficients of the migration rate, $\kappa$ denotes the local curvature of the wound edge and $H$ represents the Heaviside 
function, defined as $H(x)=0$ if $x<0$ and $H(x)=1$ if $x \geq 0$. Note that under this definition of the closure rate, the normal vector $\mathbf{n}$ points towards the wounded area.

\section{Tracking the wound edge}

The model, as presented here, falls within the category of moving boundary problems. The wound edge $\Gamma$, also referred to as the front position or interface, has to be determined at each time step in order to identify the various parts of the domain of computation (wounded region, active layer and outer tissue) and to solve the concentration equation (1).

In this work, we use the Level Set Method [29] to follow the evolution of the wound edge in time. The wound edge is identified as the zero level set of a continuous function $\phi$. At the initial time, we define the level set function $\phi$ as follows:

$$
\phi(\mathbf{x}, 0)= \begin{cases}+\operatorname{dist}(\mathbf{x}, \Gamma(0)), & \text { if } \mathbf{x} \in \Omega_{w}(0), \\ 0, & \text { if } \mathbf{x} \in \Gamma(0), \\ -\operatorname{dist}(\mathbf{x}, \Gamma(0)), & \text { if } \mathbf{x} \in \Omega_{u}(0),\end{cases}
$$

where we have arbitrarily chosen $\phi$ to be positive inside the wound, and negative outside. Using the total time-derivative of the level set function at the wound edge, we have that

$$
0=\frac{d}{d t} \phi_{\Gamma}=\frac{\partial \phi}{\partial t}+v_{n}\|\nabla \phi\| .
$$

This equation is only valid at the wound edge, but can be extended to the whole domain of computation if a velocity field $\mathbf{v}$ is determined satisfying the following conditions:

(i) $\mathbf{v}(\cdot, t)$ is continuous over $\Omega$, and

(ii) its normal projection, $\mathbf{v} \cdot \mathbf{n}$, equals the closure rate $v_{n}$ given in Eq. (2) at the wound edge.

We will present a simple velocity extension fulfilling constrains (i) and (ii) in the following section. For the time being, let us assume that such a velocity field $\mathbf{v}$ exists. The closure of the wound is then obtained by advection of the wound edge by means of the so-called level set equation:

$$
\frac{\partial \phi}{\partial t}+\mathbf{v} \cdot \nabla \phi=0
$$

Note that in this framework, the normal vector $\mathbf{n}$ and the curvature $\kappa$ can be computed from $\phi$ as follows:

$$
\mathbf{n}=\frac{\nabla \phi}{\|\nabla \phi\|}, \quad \kappa=\nabla \cdot \mathbf{n} .
$$


The interested reader is referred to the books by Osher and Fedkiw [28] and Sethian [34] where a detailed presentation of the method and a wide collection of applications are given.

\section{Re-initialization of $\phi$}

The level set function $\phi$ is initialized as a signed distance function. This choice enables us with a straightforward identification of the various subdomains in $\Omega$. In particular, the wounded tissue corresponds to the region of $\phi$ being positive, and the active layer is given by

$$
\Omega_{\mathrm{al}}(t)=\{\mathbf{x} \in \Omega \mid 0<-\phi(\mathbf{x}, t)<\delta(\mathbf{x}, t)\}
$$

where $\delta$ denotes the local thickness of the active layer, which may depend on the local curvature of the wound edge [44]. In order to keep accurate the representation of the active layer, the level set function should remain a signed distance function after solving the advection equation (4), at least a distance $\delta$ from its zero level set. Normally, this property is lost if time increases, and several remedies have been suggested for its repair. The most extended remedy is to solve the Eikonal equation

$$
\|\nabla \phi\|=1
$$

either iteratively $[30,42]$ or directly $[33,46]$. Iterative methods are very competitive if we wish to keep $\phi$ a distance function in a thin band of a couple of grid nodes around the interface. However, their computational cost increases as the bandwidth gets larger, and eventually, direct methods are computationally cheaper. In our case, the bandwidth depends on the active layer thickness, which may be large compared to the grid size. Therefore, we will use a direct method, the Fast Marching Method [33], to solve Eq. (5).

\section{The computational approach}

The solution of Eq. (1) is approximated by using a Finite Element Method with piecewise linear basis functions. Further, we have to deal with a moving interface and with a sharp (discontinuous) change of the EGF production across the edges of the active layer. Tracking the wound edge position in time with the Level Set Method allows us to deal in a straightforward manner with wounds of complicated geometries involving morphological changes. Supplementing the Finite Element Method with a local refinement of elements neighbouring the wound edge results into a more accurate representation of the EGF concentration and into higher grid resolutions for the computation of the closure rate in an efficient way.

As a background or fixed basis mesh we use a structured triangulation with linear elements, see Fig. 1(left) and, at each time step, the elements close to the interface are refined, see Fig. 1(center), according to a criterion that is specified below. After 

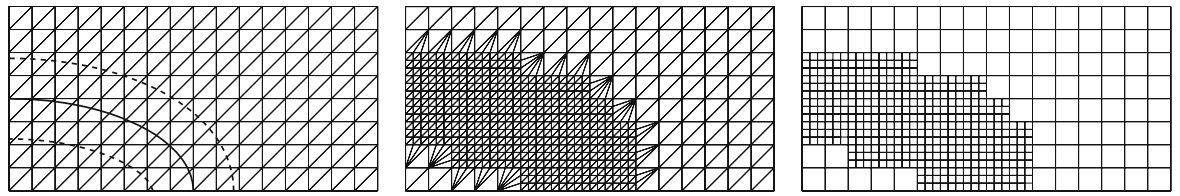

Fig. 1 Left: fixed basis FE mesh with the interface position $\phi=0$ (solid curve) and the contours $\phi= \pm d i s t$ (dashed curves). The elements within these contours are to be refined. Center: refined FE mesh. Right: the nested Cartesian grids

refinement, the refined region presents a Cartesian structure, see Fig. 1(right), where the (nonlinear) hyperbolic equations inherited from the level set formulation can be easily solved with finite difference or finite volume schemes.

Hence, in order to compute the solution (i.e. EGF concentration and wound edge location) at a certain time step, we need to perform a number of steps. Globally speaking, first we have to compute the velocity field $\mathbf{v}$ from the EGF concentration profile at the previous time step. Next, the level set function has to be updated to obtain the wound edge location at the next time step. Subsequently, $\phi$ has to be reinitialized to a distance function in order to obtain an accurate representation of the active layer, and finally, the partial differential equation (1) is solved to find the EGF distribution at the next time step. However, since the fixed basis mesh is adapted to the wound edge each time step, there are a few communication steps that have to be taken carefully. The complete algorithm is presented in Algorithm 1.

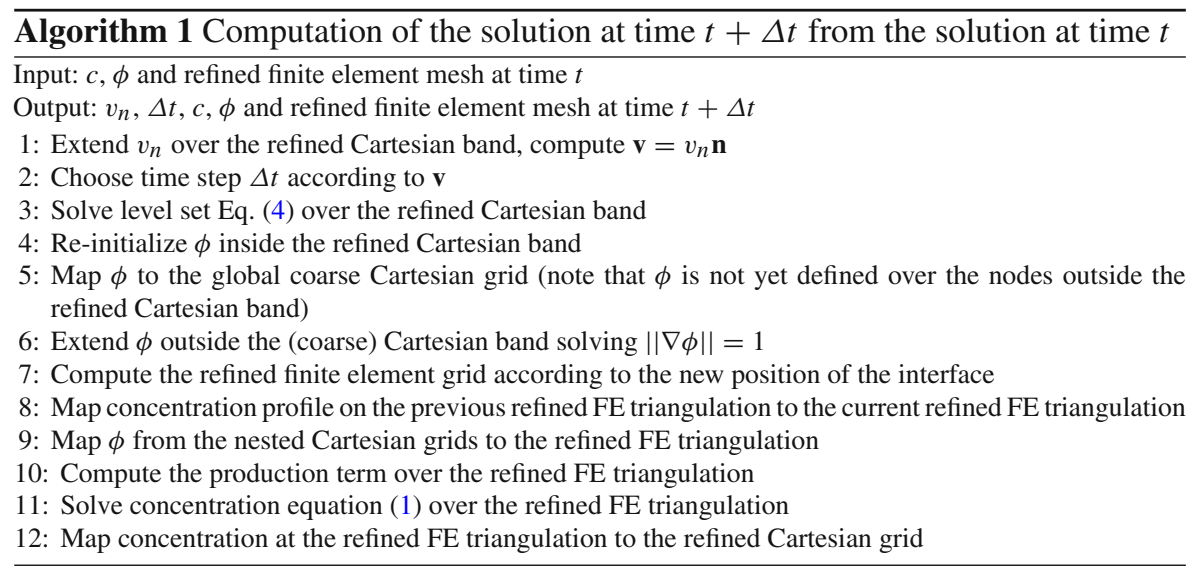

\section{Regularizations of the characteristic and Heaviside functions}

Cell motility is determined by interpolation of the EGF concentration at the wound edge. The discontinuous production term $P \chi$ is regularized in order to diminish numerical wiggles present at the edges of the active layer which may spoil the accuracy of the closure rate. Therefore, instead of the characteristic function $\chi$ we use a regularized 
version $\chi_{\varepsilon}$ defined as follows:

$$
\chi_{\varepsilon}(\phi)= \begin{cases}\frac{1}{2}\left[1+\sin \left(\frac{\pi}{\varepsilon}\left(\phi+\frac{\varepsilon}{2}\right)\right)\right], & \text { if } 0<\phi<\varepsilon, \\ 1, & \text { if } 0 \leq \phi \leq-\delta, \\ \frac{1}{2}\left[1+\sin \left(\frac{\pi}{\varepsilon}\left(\phi+\delta+\frac{\varepsilon}{2}\right)\right)\right], & \text { if }-\delta<\phi<-\delta-\varepsilon, \\ 0, & \text { otherwise, }\end{cases}
$$

where $\varepsilon$ is a parameter defined by the user to establish across how many grid cells the regularization is applied. In our numerical computations we use $\varepsilon=1.5 \cdot \max \left(\Delta x_{\mathrm{Ref}}\right.$, $\Delta y_{\text {Ref }}$, where $\Delta x_{\text {Ref }}$ and $\Delta y_{\text {Ref }}$ denote respectively the grid size of the refined band in the $x$ and $y$ directions. A similar regularization could be adopted for the definition of the closure rate, though the standard definition of the Heaviside function has been used in our calculations.

\subsection{The local grid refinement strategy}

Due to the structure of the fixed basis mesh, its refinement in the vicinity of the front position is straightforward if the level of refinement $(L O R)$ is known element-wise.

We say that an element $e$ is within a distance dist from the interface if one or more of its vertices $e_{i}$ is within a distance dist from the interface (i.e. $\left|\phi\left(e_{i}\right)\right|<d i s t$ for $i \in\{1,2,3\})$. All elements within a distance dist from the interface are labeled with the maximum LOR, that is 2 . If $d i s t=0$, elements intersected by the interface are marked with the maximum LOR. Elements adjacent to those with maximal LOR are labeled, in principle, with a lower LOR, that is 1 . However, to preserve mesh consistency, their LOR has to be increased when they are adjacent to more than one element with maximum LOR. This step is illustrated in Fig. 2. Elements further away are labeled with zero LOR, meaning that they are not refined.

The division of the elements, according to their LOR, is based on the division of the edges. Let us denote by refnratio the number of equally sized sub-edges that appear when the edge of an element is refined. If the LOR of an element is 2 , then all the edges are divided into refnratio sub-edges, and the element is divided into ref nratio $o^{2}$ sub-elements. If the LOR is 1 , only the edge in common with the neighbouring element with LOR equal to 2 is refined. Consequently, the element is divided into refnratio sub-elements (see Fig. 2).

In order to prevent excessively narrow elements, which may impair the accuracy of the results, we will restrict ourselves to ratios of refinement, refnratio, of 2 or 3 . To balance the additional work invested in the refinement with the gain in accuracy and

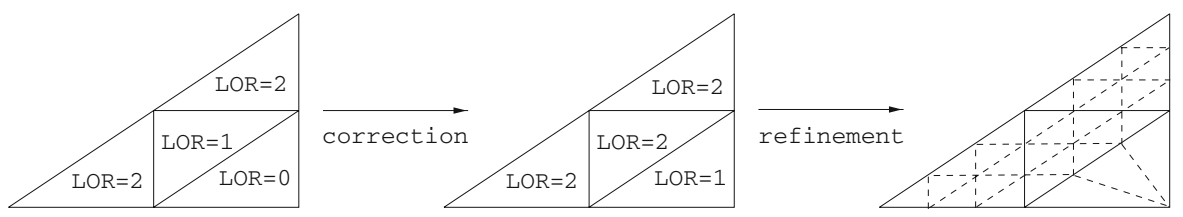

Fig. 2 Correction of the LOR and subsequent subdivision of the elements, with ref nratio $=3$ 
CPU-times, we define dist such that the elements intersected by the interface and the elements adjacent to them receive the maximum LOR. Hence, dist $\gtrsim \sqrt{\Delta x^{2}+\Delta y^{2}}$, where $\Delta x$ and $\Delta y$ denote the element size of the basis mesh. Furthermore, we shall impose a maximum displacement bound on the time stepping to prevent that the level of refinement of elements close to the interface changes by more than one within two consecutive time steps. As we will see below, this is ensured by a CFL condition on the advection of the level set function.

The transition between two consecutive refined grids (i.e. two consecutive time steps) can be implemented efficiently if the LOR of the previous mesh is stored in memory. By construction, the LOR of an element at the new time step either does not change, or increases by one, or reduces by one its LOR at the previous time step. If the LOR does not change, the refinement of the element is the same as in the previous time step. If the LOR is reduced by one, the element is coarsen. Finally, if the LOR is raised by one, then the refinement of the element increases.

\subsection{The two nested Cartesian grids}

Due to its structure, the nodes of the background triangulation will be referred to as the coarse Cartesian grid. Moreover, after refinement, the refined region resembles a refined Cartesian band which is nested in the coarse Cartesian grid, see Fig. 1(right). We use both grids to solve the hyperbolic equations inherited from the level set formulation.

\subsubsection{The narrow band level set method}

In order to update the front position we first need to compute an extension of the front velocity, and subsequently solve the level set Eq. (4). These operations are carried out within the refined Cartesian band.

\section{Extension of the front velocity off the interface}

The front velocity $v_{n}$ is only defined at the interface location, and an artificial extension of it is required to solve the level set Eq. (4). As stated before, this extension should be such that the extended velocity field is continuous. To achieve this, we advect the front velocity outwards in the proper upwind direction, solving

$$
\frac{\partial v_{n}}{\partial \tau}+\mathrm{S}(\phi) \mathbf{n} \cdot \nabla v_{n}=0
$$

where $\tau$ denotes a fictitious time used only in the extension procedure and $\mathbf{n}$ denotes the normal vector, which points towards increasing values of $\phi$ (i.e. in the direction of the gradient of $\phi$, and note that $\phi>0$ inside the wound). Thus, with this equation, information is transported from the wound edge inside the wound (when $\phi>0$ ) and outside the wound (when $\phi<0$ ). Note that at the wound edge, the previous equation is degenerated, since $\mathrm{S}(\phi)=0$, and hence the value of $v_{n}$ is not altered. 
The solution of Eq. (6) is computed only inside the refined Cartesian band, as described in Algorithm 2. We perform a small number of pseudo-time iterations Iter Max, between 5 and 10, since it is enough to obtain a continuous extension of $v_{n}$. The normal vector $\mathbf{n}=\nabla \phi /\|\nabla \phi\|$ is approximated with central differences inside the band and first order sided differences at the boundary. The local curvature $\kappa=\nabla \cdot \mathbf{n}$ is computed analogously.

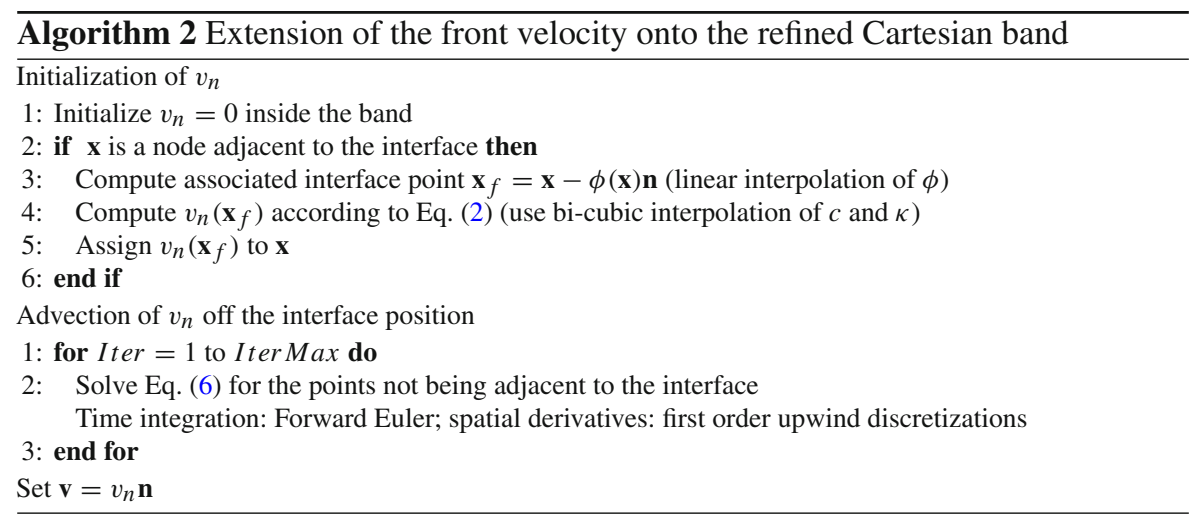

\section{Interface advection and re-initialization of $\phi$ to a distance function}

After the advection field $\mathbf{v}=(u, v)^{t}$ is defined over the band, the level set Eq. (4) is solved inside the band. The time integration is carried out with the Euler forward method, whereas the space derivatives are discretized with a first order accurate upwind scheme. The CFL stability condition is satisfied if the time step $\Delta t$ is chosen such that

$$
\Delta t \leq \Delta t_{\mathrm{CFL}}:=\frac{\mathrm{CFL}}{\max \left(\frac{u}{\Delta x}+\frac{v}{\Delta y}\right)}, \quad \mathrm{CFL}<1 .
$$

In practice, we will take $\Delta t=\min \left(\Delta t_{\mathrm{CFL}}, \Delta t_{\max }\right)$, where $\Delta t_{\max }$ is used as a default time step during the incubation periods and prevents the use of excessively large time steps when the front velocity is close to zero. With this choice of $\Delta t$, the wound edge will travel over a distance smaller than the grid size during each time step, which is consistent with the grid refinement algorithm.

Despite that the level set function $\phi$ is initialized as a distance function, the function $\tilde{\phi}$ resulting from the solution of Eq. (6) is no longer a distance function. Generally, its deviation after one time step is small, and re-initialization may be evitable. However, the error accumulates, and after several time steps re-initialization is unavoidable. In our case, we use a second order accurate Fast Marching Method to solve the Eikonal equation $\|\nabla \phi\|=1$ inside the band. This method proceeds as follows. First, the function $\phi$ is initialized at the nodes adjacent to the interface in such a way that the zero level set of $\tilde{\phi}$ is not altered. Subsequently, $\phi$ is extended away in the downwind direction. Details of this method can be found in [33]. 


\subsubsection{Extension of $\phi$ outside the band}

In order to extend $\phi$ further away, i.e. outside the refined Cartesian band, we first map it to the coarse Cartesian band (i.e. to the nodes which lay both in the refined band and in the coarse global grid) and subsequently we extend $\phi$ outside the band with the Fast Marching Method. As a result, we obtain a function $\phi$ that is defined on the basis mesh, which does not alter the interface position, and which is a distance function.

\section{Asymptotic solutions}

This section is devoted to study two simplified problems. In the first case, we consider the healing process in absence of transport of the EGF, which prevents the supply of EGF to the wound site. In the second case, we look at the healing process with instantaneous diffusion of the EGF, which provides the wound with a suboptimal EGF distribution. By analysis of their analytic solutions, we will give valuable insights to the healing behaviour predicted by the model and start to discern the role of some parameters in the model.

We define $t_{\text {inc }}$ as the incubation time that is necessary to activate the closure of the wound. Hence, $t_{\mathrm{inc}}$ is the smallest positive time at which $c\left(\mathbf{x}, t_{\mathrm{inc}}\right) \geq \theta$ for any point $\mathbf{x}$ on the initial wound edge. After this time, the healing process starts and the wound size decreases. However, new storage or incubation periods may become necessary if the EGF level at the wound edge falls below $\theta$. Hence, we can describe the healing process as a series of healing and incubation periods as sketched in Fig. 3, where $\mathcal{I}_{\text {heal }}^{i}$ $(i=1,2, \ldots)$ denotes the healing period within the consecutive incubation periods $\mathcal{I}_{\text {inc }}^{i-1}$ and $\mathcal{I}_{\text {inc }}^{i}$. By convention we denote by $t_{\text {inc }}^{i}$ and $t_{\text {heal }}^{i}$ the times at which $\mathcal{I}_{\text {inc }}^{i}$ and $\mathcal{I}_{\text {heal }}^{i}$ finish respectively, which are formally defined as

$$
\begin{aligned}
t_{\text {heal }}^{i} & :=\min \left(t>t_{\text {inc }}^{i-1} \mid c(\mathbf{x}, t)<\theta \text { for all } \mathbf{x} \in \Gamma(t)\right), \quad i \geq 1, \\
t_{\text {inc }}^{i} & :=\min \left(t>t_{\text {heal }}^{i} \mid c(\mathbf{x}, t) \geq \theta \text { for any } \mathbf{x} \in \Gamma\left(t_{\text {heal }}^{i}\right)\right), \quad i \geq 1 .
\end{aligned}
$$

We extend this convention by denoting $\mathcal{I}_{\text {inc }}^{0}$ the first or initial incubation period, $t_{\text {inc }}^{0}=$ $t_{\text {inc }}$ and $t_{\text {heal }}^{0}=0$. For sake of the presentation, we will refer to $\mathcal{I}_{\text {inc }}^{i}$ for $i \geq 1$ as internal incubation periods.

\subsection{No diffusional transport of the EGF}

If we assume that there is no diffusional transport of the EGF from the active layer towards the wound, i.e. $D=0$, then the whole production of EGF is maintained inside the active layer and no EGF flows into the wound. Hence, during a whole incubation period $\mathcal{I}_{\text {inc }}^{i}$, the EGF concentration is given by

$$
\frac{\partial c}{\partial t}+\lambda c=P \chi_{\Omega_{\mathrm{al}}\left(t_{\text {heal }}^{i}\right)},
$$


Fig. 3 Schematic diagram of the healing process

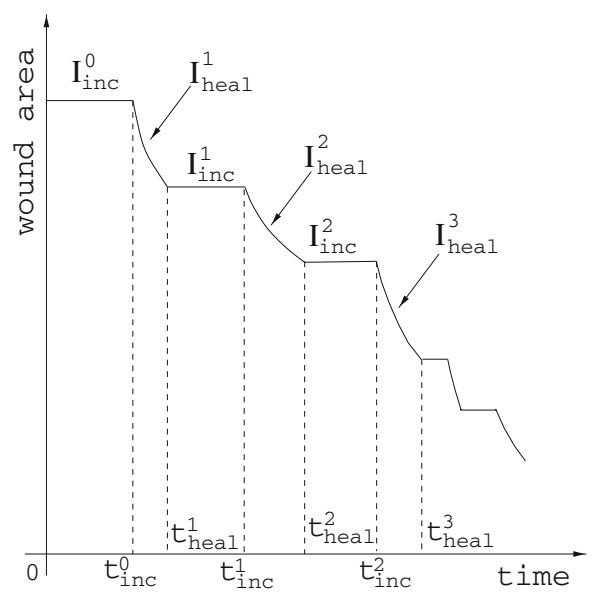

with initial condition the concentration at the beginning of the incubation period, i.e. $c\left(\mathbf{x}, t_{\text {heal }}^{i}\right)$. Integration in time of the above equation yields:

$c(\mathbf{x}, t)=\exp \left(-\lambda\left(t-t_{\text {heal }}^{i}\right)\right) c\left(\mathbf{x}, t_{\text {heal }}^{i}\right)+\frac{P}{\lambda} \chi_{\Omega_{\text {al }}\left(t_{\text {heal }}^{i}\right)}(\mathbf{x})\left(1-\exp \left(-\lambda\left(t-t_{\text {heal }}^{i}\right)\right)\right)$,

for $\mathbf{x} \in \Omega$ and $t \in \mathcal{I}_{\text {inc }}^{i}$. Moreover, since the EGF production is not diffused towards the wound from the active layer, the concentration at the wound edge decays to zero as soon as it moves and hence $c\left(\mathbf{x}, t_{\text {heal }}^{i}\right)=0$ for all $\mathbf{x} \in \Gamma\left(t_{\text {heal }}^{i}\right)$. Therefore, the concentration at the wound edge is given by

$$
c(\mathbf{x}, t)=\frac{P}{\lambda}\left(1-\exp \left(-\lambda\left(t-t_{\text {heal }}^{i}\right)\right)\right), \quad \text { for } \mathbf{x} \in \Gamma\left(t_{\text {heal }}^{i}\right), \quad t \in \mathcal{I}_{\text {inc }}^{i}
$$

and the healing process will be initiated or resumed within a finite time if and only if

$$
\lim _{t \rightarrow \infty} \frac{P}{\lambda}\left(1-\exp \left(-\lambda\left(t-t_{\text {heal }}^{i}\right)\right)\right)>\theta
$$

i.e. if and only if $P / \lambda>\theta$. This condition was also found by Adam [3] using the analytic solution of the steady-state problem, and manifests the necessity for the production inside the active layer to overcome the chemical depletion as the concentration reaches the threshold level $\theta$. It is worth noting that, in the case of no diffusion of the EGF, the thickness or shape of the active layer does not play any role.

Finally, if the healing process can be sustained, i.e. $P / \lambda>\theta$, then the initial incubation time $t_{\text {inc }}$ is given by

$$
t_{\mathrm{inc}}^{D=0}=\frac{1}{\lambda} \log \left(\frac{1}{1-\frac{\lambda \theta}{P}}\right) .
$$




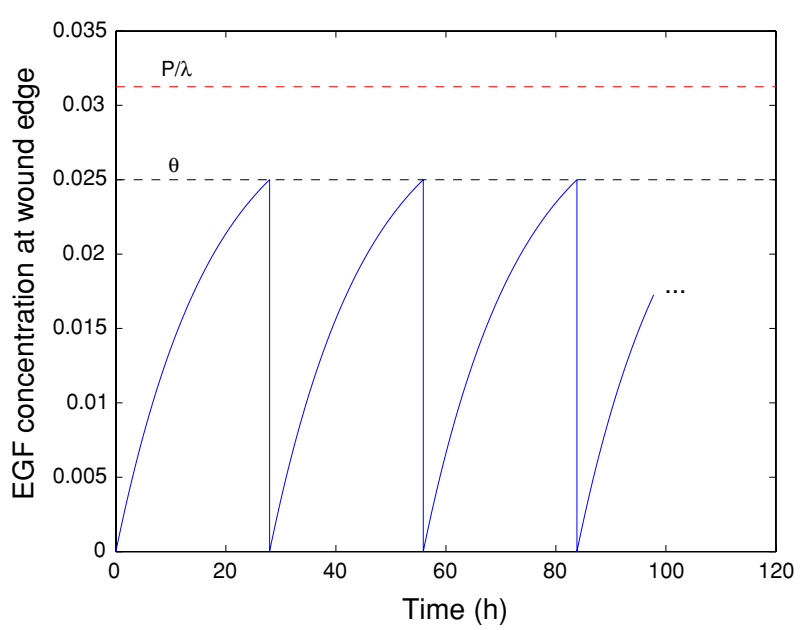

Fig. 4 Time progression of the EGF concentration at the edge of a circular wound for the case of $D=0$. The horizontal line indicates the upper bound for the threshold value $\theta$ in order to initiate healing

Furthermore, because the closure of the wound is permanently interrupted to restore the EGF concentration at the wound edge, healing of the wound will just not occur. The EGF concentration at the edge of a circular wound during the first three incubation periods is plotted in Fig. 4, where the 'broken' healing behaviour of the solution can be observed.

\subsection{Instantaneous transport of the EGF}

In the case of an instantaneous diffusion of the EGF, the production inside the active layer is immediately distributed throughout the whole domain of computation and the EGF concentration shows a flat profile over time. Hence, by integration of Eq. (1) over $\Omega$, we can define an effective production rate

$$
P_{\text {eff }}(t):=P \frac{\left|\Omega_{\mathrm{al}}(t)\right|}{|\Omega|},
$$

where $|\mathcal{A}|$ denotes the area of $\mathcal{A}$. With this definition, the EGF concentration is given by the solution of

$$
\frac{d c}{d t}+\lambda c=P_{\mathrm{eff}}(t)
$$

with initial condition $c(0)=0$. The definition of $P_{\text {eff }}$ indicates the relevance of the area of the active layer in the healing process. For instance, if the thickness of the active layer is kept constant during healing, then the area of $\Omega_{\mathrm{al}}$ decreases and hence the production sustained by it diminishes as the wound heals. Despite this seems adequate since the wound area decreases, it may result in an incomplete healing of the wound as we will show below. 
Exact integration of Eq. (10) is not always possible since $P_{\text {eff }}$ depends on the evolution of the wound. However, it can be solved during the incubation periods, since then $P_{\text {eff }}$ is constant. Thus, the EGF concentration during the $i$ th incubation period $\mathcal{I}_{\text {inc }}^{i}$ is given by

$$
\begin{gathered}
c(t)=\frac{P_{\text {eff }}(0)}{\lambda}(1-\exp (-\lambda t)), \quad \text { for } i=0, \\
c(t)=\exp \left(-\lambda\left(t-t_{\text {heal }}^{i}\right)\right) \theta+\frac{P_{\text {eff }}\left(t_{\text {heal }}^{i}\right)}{\lambda}\left(1-\exp \left(-\lambda\left(t-t_{\text {heal }}^{i}\right)\right)\right), \quad \text { for } i \geq 1 .
\end{gathered}
$$

Because of the continuity of $c$, it is easy to see that the healing process will be initiated or continued if and only if

$$
\frac{P_{\text {eff }}\left(t_{\text {heal }}^{i}\right)}{\lambda}>\theta, \quad \text { for } i \geq 0, \quad \Longleftrightarrow \frac{P}{\lambda} \frac{\left|\Omega_{\text {al }}\left(t_{\text {heal }}^{i}\right)\right|}{|\Omega|}>\theta, \quad \text { for } i \geq 0 \text {. }
$$

Hence, in the case of instantaneous transport of the EGF, the healing process will start if and only if

$$
\frac{P}{\lambda}>\theta \frac{|\Omega|}{\left|\Omega_{\mathrm{al}}(0)\right|},
$$

which, for fixed decay rate $\lambda$ and threshold concentration $\theta$, provides a lower bound for the production rate $P$ as function of the active layer and the animal sizes. When the healing process can be initiated, the initial incubation time is given by

$$
t_{\mathrm{inc}}^{D \rightarrow \infty}=\frac{1}{\lambda} \log \left(\frac{1}{1-\frac{\lambda \theta}{P} \frac{|\Omega|}{\left|\Omega_{\mathrm{al}}(0)\right|}}\right) .
$$

Note furthermore that initiation of the healing process is much more demanding in the case of fast diffusion processes, as can be seen by comparison of Eqs. (8) and (11), since it is reasonable to expect that $|\Omega| /\left|\Omega_{\mathrm{al}}(0)\right| \gg 1$. Moreover, if diffusion is instantaneous (i.e. $D \rightarrow \infty$ ), healing will not be resumed once it ceased due to an insufficient size of the active layer. The reason is that this insufficient size gives $c^{\prime}\left(t_{\text {heal }}^{i}\right)<0$, making impossible the accumulation of the necessary growth factor at the wound edge.

\section{Healing response of a circular wound under instantaneous transport of the EGF}

The analytic expression of the EGF concentration during healing can be obtained for certain definitions of the active layer and the mitotic rate. In the remaining of this section we consider the healing response of a circular wound of radius $r_{0}$ in a circular domain of computation of radius $R_{c}$ and an active layer of constant thickness $\delta$. In this case, the area of the active layer is given by

$$
\left|\Omega_{\mathrm{al}}(t)\right|=\pi \delta(2 r(t)+\delta)
$$


Table 1 Description of the healing response as function of the physiological parameters of the model

\begin{tabular}{lll}
\hline$\theta$ & $\alpha$ & Healing \\
\hline$\leq \frac{P}{\lambda} \frac{\delta^{2}}{R_{c}^{2}}$ & Any & Complete \\
$\in\left(\frac{P}{\lambda} \frac{\delta^{2}}{R_{c}^{2}}, \frac{P}{\lambda} \frac{\delta\left(r_{0}+\delta\right)}{R_{c}^{2}}\right]$ & Large & Complete \\
$\in\left(\frac{P}{\lambda} \frac{\delta\left(r_{0}+\delta\right)}{R_{c}^{2}}, \frac{P}{\lambda} \frac{\delta\left(2 r_{0}+\delta\right)}{R_{c}^{2}}\right]$ & Small & Incomplete \\
$>\frac{P}{\lambda} \frac{\delta\left(2 r_{0}+\delta\right)}{R_{c}^{2}}$ & Any & Incomplete \\
\hline
\end{tabular}

where $r(t)$ denotes the wound radius at time $t$. If in addition we take $\beta=0$, then

$$
r(t)=r_{0}-\alpha\left(t-t_{\text {inc }}\right),
$$

and

$$
\begin{aligned}
c(t)= & \theta \exp \left(-\lambda\left(t-t_{\text {inc }}\right)\right)+\frac{P \delta}{R_{c}^{2} \lambda^{2}}\{\lambda(2 r(t)+\delta) \\
& \left.+2 \alpha-\exp \left(-\lambda\left(t-t_{\text {inc }}\right)\right)\left(\lambda\left(2 r_{0}+\delta\right)+2 \alpha\right)\right\},
\end{aligned}
$$

during the first healing period, i.e. $t \in \mathcal{I}_{\text {heal }}^{1}$. The complete healing of the wound occurs if and only if there is a time $t_{\text {heal }}>t_{\text {inc }}$ such that $r\left(t_{\text {heal }}\right)=0$ and $c\left(t_{\text {heal }}\right)>\theta$. It is easy to see, after simple algebraic manipulations, that this condition is equivalent to

$$
\theta<\frac{P \delta}{R_{c}^{2} \lambda^{2}} \frac{2 \alpha+\lambda \delta-\exp \left(-\frac{\lambda r_{0}}{\alpha}\right)\left(2 \alpha+\lambda\left(2 r_{0}+\delta\right)\right)}{1-\exp \left(-\frac{\lambda r_{0}}{\alpha}\right)}
$$

which establishes the healing success as a function of the physiological parameters of the model. Table 1 summarizes the healing response for several values of $\theta$ and $\alpha$.

For the cases in which the healing is not complete, the wound radius at the stopping time can be computed from Eq. (13), since then there is a time $t_{\text {stop }}>t_{\text {inc }}$ such that $c\left(t_{\text {stop }}\right)=\theta$ and $r_{\text {stop }}:=r_{0}-\alpha\left(t_{\text {stop }}-t_{\text {inc }}\right)$, which yields

$$
\theta=\frac{P \delta}{R_{c}^{2} \lambda^{2}} \frac{2 \alpha+\lambda\left(2 r_{\text {stop }}+\delta\right)-\exp \left(-\lambda \frac{r_{0}-r_{\text {stop }}}{\alpha}\right)\left(2 \alpha+\lambda\left(2 r_{0}+\delta\right)\right)}{1-\exp \left(-\lambda \frac{r_{0}-r_{\text {stop }}}{\alpha}\right)} .
$$

The EGF concentration during time is plotted in Fig. 5a for two different values of $\theta$, of which the smallest gives complete healing of the wound whereas the largest yields incomplete healing. The healing response and wound radius at stop for a wide range 

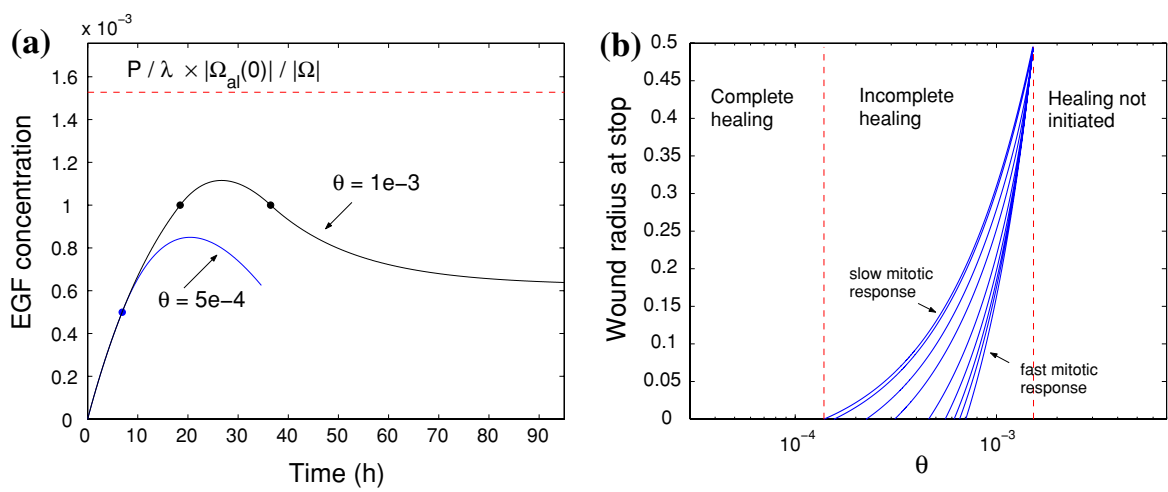

Fig. 5 Healing of a circular wound with instantaneous diffusion of the EGF. a Time evolution of the EGF concentration for two different values of $\theta$. The horizontal line indicates the upper bound for $\theta$ in order to initiate healing; b The healing response in the case of $D \rightarrow \infty$ for several closure rates. Under this notation, successful healing is obtained when $r_{\text {stop }}=0$

of closure rates is presented in Fig. 5b, where the reduction of $r_{\text {stop }}$ towards successful healing (i.e. $r_{\text {stop }}=0$ ) by the increase of the migration rate can be observed.

\section{Healing time}

If healing is complete (see Table 1), then it proceeds directly after incubation without internal incubation times. Hence, the wound evolution after initiation is the same as if the threshold condition upon cell motility is disregarded or $\theta$ is set equal to zero. Therefore,

$$
t_{\text {heal }}^{D \rightarrow \infty}=t_{\text {inc }}^{D \rightarrow \infty}+t_{\text {heal }}^{\theta=0} .
$$

Computation of $t_{\text {heal }}^{\theta=0}$ for general wound morphologies only requires the solution of Eq. (4) with $v_{n}=\alpha+\beta \kappa$, which is much easier than the solution of the complete problem. Furthermore, $t_{\text {heal }}^{\theta=0}$ is known for simple wound morphologies [44].

\section{Analysis of healing kinetics}

The specific role of diffusion and cell migration rates in the healing behaviour described by Eqs. (1), (2) and (4) are discussed here. Results for a circular wound under various physiological conditions will show the strong interdependence between these processes and their impact on the healing kinetics. Results for more complex wound morphologies evidence locally different healing behaviours.

\subsection{The role of diffusion in the healing kinetics}

The diffusion term in Eq. (1) is responsible for the supply of EGF to the wound in order to sustain its healing. We have seen in Sect. 4 that in absence of diffusion, the 

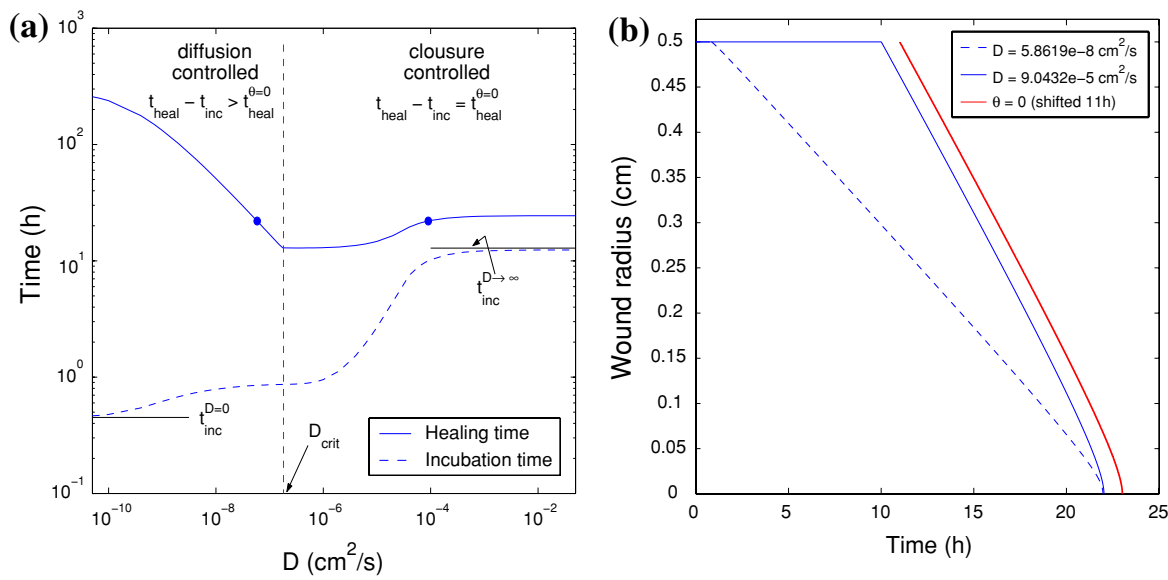

Fig. 6 Influence of the diffusion term in the healing behaviour. a Incubation and healing times as a function of $D$. b Healing behaviour for two different values of $D$ that give the same healing time

incubation time $t_{\text {inc }}$ is minimal but healing is not obtained in a finite time. On the other hand, if diffusion is instantaneous, the incubation time is exponentially increased by the ratio of the domain of computation and the active layer areas, i.e. $|\Omega| /\left|\Omega_{\mathrm{al}}(0)\right|$. Furthermore, in the case of successful healing, it proceeds directly after incubation.

The incubation and healing times for a wide range of diffusion rates $D$ are plotted in Fig. 6a. The incubation time continuously increases as the diffusion rate increases, as the EGF is sent far from the interface delaying its accumulation. Furthermore, the results for the incubation times as $D \rightarrow 0$ and $D \rightarrow \infty$ agree perfectly with the asymptotic values given in Eqs. (9) and (12) respectively. Besides that, we observe that the healing time increases exponentially as the diffusion rate decreases, since then the closure of the wound is faster than the EGF diffusion into the wound. This implies, numerically, more and longer internal incubation times, and physiologically the impossibility to sustain the healing process. As $D$ increases, the healing time reduces until it reaches its minimum. This minimal healing time is obtained when the diffusion and the closure rates are balanced. We denote by $D_{\text {crit }}$ the critical diffusion rate that gives the minimal healing time. We observe that the difference between the healing and the incubation times remains constant for diffusion rates above $D_{\text {crit }}$, and the time consumed between incubation and healing agrees with the healing time of the wound with $\theta=0$. This means that if $D>D_{\text {crit }}$, then the healing progress is not delayed by internal incubation times and therefore the increase of the healing time is entirely due to the increase of the incubation time. Furthermore, in these cases the closure of the wound is slower than the diffusion of the EGF into the wound.

As a consequence of the behaviour of the healing time with respect to the diffusion rate, there exist pairs of diffusion rates that result in the same healing time. The evolution of the wound radius for one of these pairs is presented in Fig. 6b. As is to be expected, the healing at the lower diffusion rate is initiated earlier. However, for the higher diffusion rate, the evolution of the wound radius after initiation is the same as for $\theta=0$, whereas it is delayed for the lower diffusion rate. 

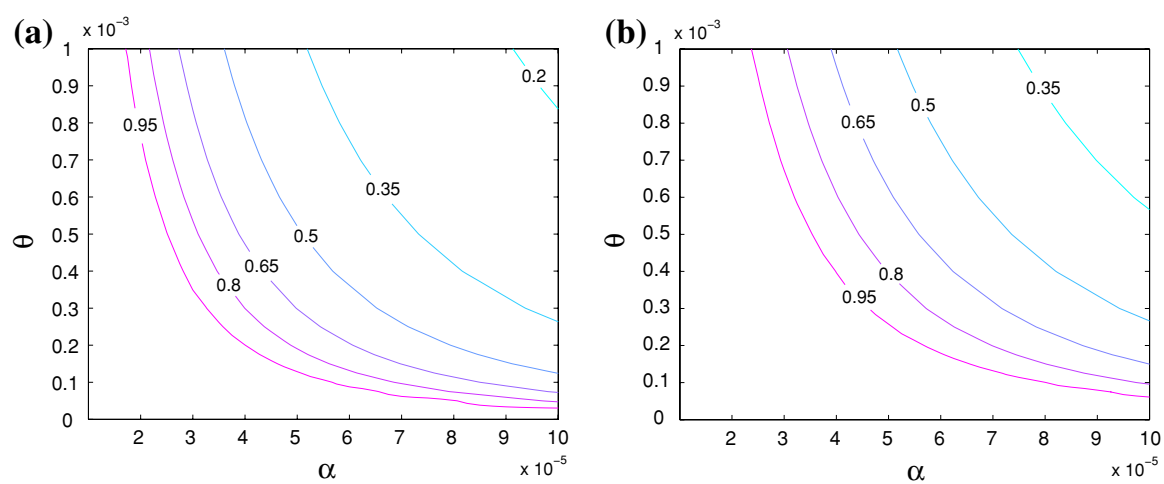

Fig. 7 Isocurves of the delay factor $f_{\text {delay }}$ as a function of the closure rate $\alpha$ and the threshold concentration $\theta$ for two diffusion rates. The contribution of the curvature to the closure rate has been disregarded, i.e. $\beta=0$. Hence, the healing time for $\theta=0$ is given by $t_{\text {heal }}^{\theta=0}=\frac{r_{0}}{\alpha} \cdot \mathbf{a} D=5 \cdot 10^{-7} \mathrm{~cm}^{2} / \mathrm{s} . \mathbf{b} D=10^{-6} \mathrm{~cm}^{2} / \mathrm{s}$

\subsection{The role of the closure rate in the healing kinetics}

In the previous section we have seen that the healing process is optimal, in the sense that it is not delayed, if the wound closure is slower than the diffusion of the EGF into the wound. In this section, we will measure the delay of the healing process due to the closure rate.

The delay in the healing process can be measured by

$$
t_{\text {delay }}=\left(t_{\text {heal }}-t_{\text {inc }}\right)-t_{\text {heal }}^{\theta=0}
$$

which primarily depends on the threshold condition $\theta$ and on the migration rate parameters $\alpha$ and $\beta$. Furthermore, we can define a delay or retardation factor as follows:

$$
f_{\text {delay }}:=\frac{t_{\text {heal }}^{\theta=0}}{t_{\text {heal }}-t_{\text {inc }}},
$$

which equals 1 if there is no delay and is smaller than 1 if there is some delay. Hence, the healing kinetics for any threshold condition $\theta>0$ can be expressed as a delay of the healing kinetics for $\theta=0$, since $t_{\text {heal }}=t_{\text {inc }}+t_{\text {heal }}^{\theta=0} / f_{\text {delay. The delay factor for a }}$ circular wound as a function of $\theta$ and $\alpha$ is presented in Fig. 7 for two different diffusion rates. The contribution to the closure rate of the wound curvature $\beta$ is disregarded in this test, i.e. $\beta=0$. The results show that for either low migration rates or $\theta$ values no delay is obtained. However, there is an exponential decrease of the delay factor as $\alpha$ and $\theta$ increase, since then the diffusion term is not capable of sustain the necessary flux of EGF into the wound. Furthermore, comparison of the results for the two different diffusion rates shows that a larger diffusivity mitigates the delayed kinetics for a wider range of $\theta$ and $\alpha$. This agrees with the results obtained in the previous section. 

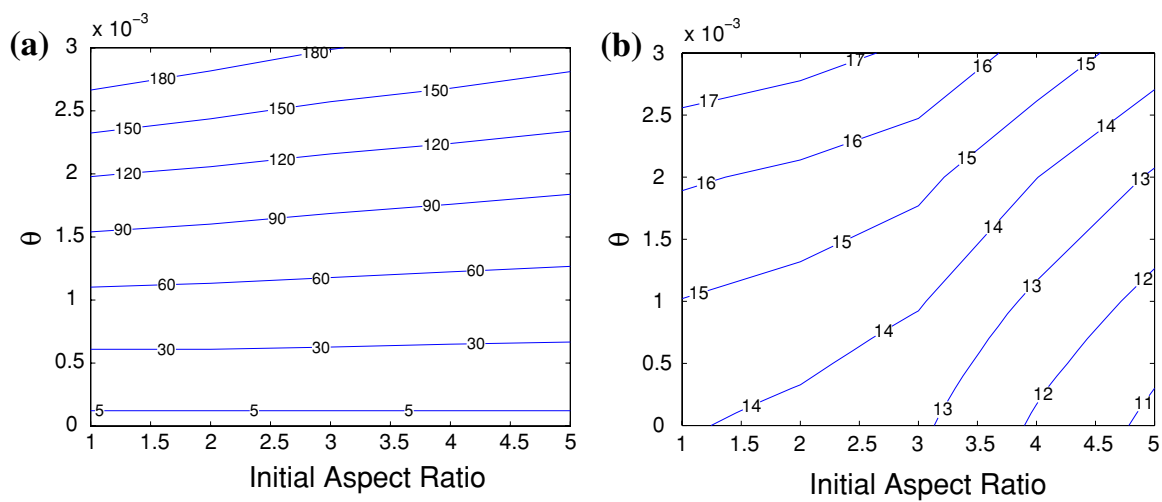

Fig. 8 Influence on the healing behaviour of the wound morphology and $\theta$. In all the cases, the initial area of the wound is the same. a Incubation times (in minutes) for several elliptical wounds and $\theta$ values. b Healing times (in hours) for several elliptical wounds and $\theta$ values

\subsection{The effect of wound geometry on healing kinetics}

The role of wound morphology on the healing kinetics is investigated in this section. To this end, we start with the healing of an elliptical wound of area $0.78540 \mathrm{~cm}^{2}$ with various elongations. In this simulation, we neglect the mechanical forces acting on the tissue. Due to the symmetry of the problem, we solve only one quarter of the ellipse. The incubation times for various wound elongations and $\theta$ values are presented in Fig. 8a. It can be seen from the results that the incubation time is reduced by the elongation of the wound, although this effect is only significant for large values of $\theta$. This is a consequence of the diffusion term, which aids the accumulation of the EGF at the regions of high curvature. As is to be expected, we observe an exponential increase of the incubation time with $\theta$. The isocurves of the healing time for various wound elongations and $\theta$ values are plotted in Fig. $8 \mathrm{~b}$. Similarly to the incubation time, the healing time increases with $\theta$ and decreases with the wound elongation. However, the increase of the healing time with $\theta$ is merely linear, since the EGF accumulation inside the wound reduces the retardation of the healing kinetics.

Finally, we consider the healing of a wound with a more complicated geometry for various values of $\theta$ and $\beta$. The initial shape of the wound is a four-legged starfish whose edge is given by the zero level set of the function $\phi=\max \left(\phi_{s}, \phi_{c}\right)$, where $\phi_{s}$ denotes the distance function whose zero level set is the curve

$$
x=\left(a+b \cos \left(2 n_{\text {legs }} \pi \varphi\right) \cos (2 \pi s), \quad y=\left(a+b \cos \left(2 n_{\text {legs }} \pi \varphi\right) \sin (2 \pi s)\right.\right.
$$

where $\varphi \in[0,1]$ and $n_{\text {legs }}$ denotes the number of legs of the starfish, four in this case, and $\phi_{c}$ denotes the distance function whose zero level set is the circle of radius $a-\frac{4}{6} b$ and centered at the origin. By construction, $\phi_{s}$ and $\phi_{c}$ must be chosen positive at the origin, in order to have $\phi$ positive inside the wound.

The initial wound together with the morphology when 30, 60 and $90 \%$ of the initial area is healed are presented in Fig. 9. This morphology, although hardly found in practice, illustrates nicely several aspects of the model. First, that the contribution 


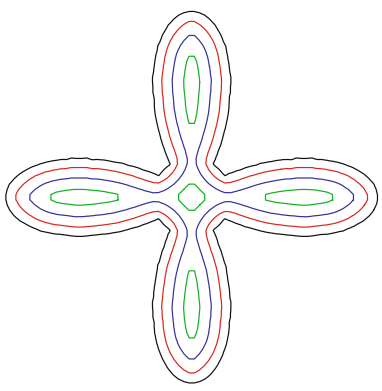

(a)

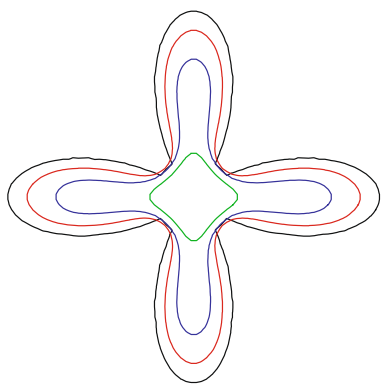

(d)

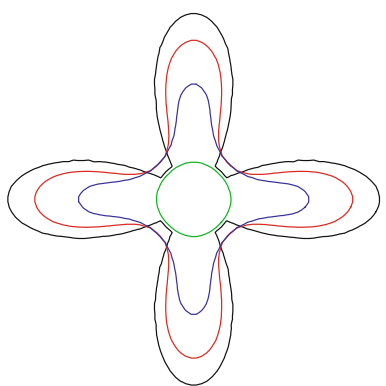

(g)

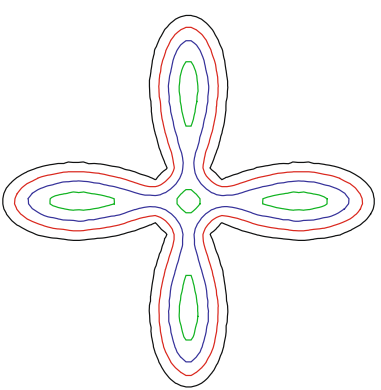

(b)

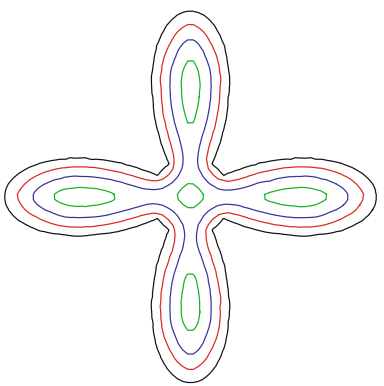

(e)

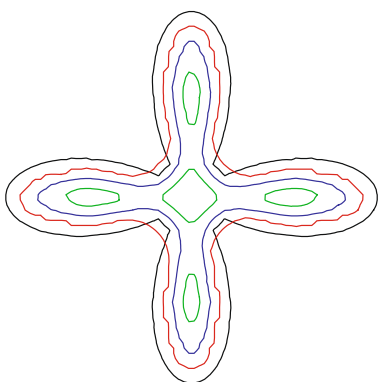

(h)

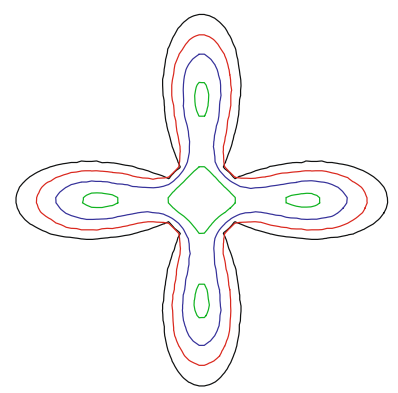

(c)

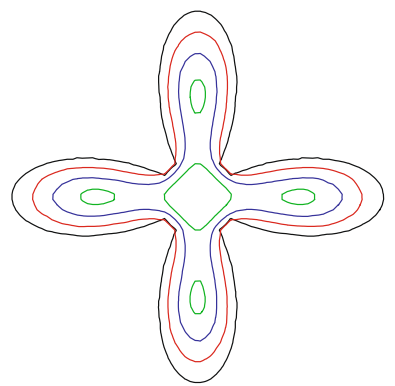

(f)

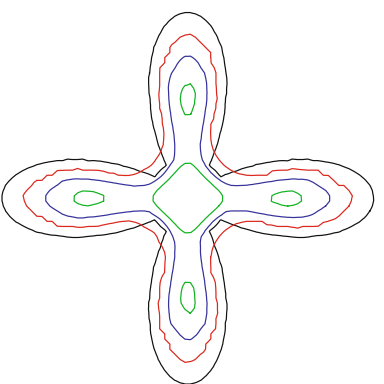

(i)

Fig. 9 Effect of physiological parameters on the morphology of the wound during healing. a $\alpha=1, \beta=0$, $\theta=0, \mathbf{b} \alpha=1, \beta=0, \theta=10^{-4}, \mathbf{c} \alpha=1, \beta=0, \theta=10^{-3}, \mathbf{d} \alpha=1, \beta=0.05, \theta=0$ e $\alpha=1$, $\beta=0.05, \theta=10^{-4}$, f $\alpha=1, \beta=0.05, \theta=10^{-3}, \mathbf{g} \alpha=1, \beta=0.1, \theta=0, \mathbf{h} \alpha=1, \beta=0.1$, $\theta=10^{-4}, \mathbf{i} \alpha=1, \beta=0.1, \theta=10^{-3}$

of the curvature to the migration rate may induce a retreat of the wound edge in the concave regions, see Fig. 9d, g, h, i. Second, that larger values of $\theta$ results into longer lag periods for the concave parts of the wound. However, once initiated, the healing of the concave areas is more easily sustained because of the EGF accumulation due to the overlap of the diffusion fronts. And last, that during healing the wound may evolve into several (five in this case) disjoint smaller wounds that heal as isolated islands. We have obtained this behaviour for small values of $\beta$ or large values of $\theta$, see Fig. 9a-c, e, f, h, i. 


\section{Discussion}

A computational model for the simulation of epidermal wound healing, with special attention to wound morphology, has been proposed in this work. The solution approach is based on the well known Level Set Method, which allows for complex evolutions of the wound edge. This method has been used in a wide range of applications, from fluid dynamics [42], materials science [19] to computer vision [21]. It has been recently applied to tumour growth [18] and, as far as we know, the present work is the first time it is applied to model wound healing dynamics. Local refinement of the computational grid around the wound edge is applied to capture the healing kinetics more accurately and efficiently, exploiting the combination of finite element and finite difference/finite volume methods. Healing is induced by EGF production by cells surrounding the wound, and a switch closure mechanism based on a threshold EGF concentration is used to determine the regions of actual healing as time progresses [3].

The current mathematical model treats the wound edge specifically, unlike other models. The healing kinetics are phenomenologycal and cope with the chemical control of re-epithelialization in a rather simple fashion. However, the healing behaviour calculated from it agrees qualitatively well with existing models [37] (not shown). Moreover, it is worth noting that in the present model, cell population is not incorporated (which may be seen as a deficiency, since for instance chemotaxis may not be included) and only the position of the wound edge is really considered. Strictly speaking, the trajectory of cells invading the wound site is not relevant (to the model), and only the point at which they contribute to healing is of interest (to the model). Two closure kinetics are predicted by the simulations (see Sects. 5.1 and 5.2): a delayed cellular migration, when the theoretical closure rate cannot be achieved because of insufficient supply of EGF to the wound edge, and a 'geometric' cellular migration, when the EGF supply is guaranteed after the incubation period and the wound heals as predicted by its geometry.

As in most of the published models (and in all the cited here), we see the wound from the top, and disregard many important aspects related to its depth. Nevertheless, deep wounds heal differently to superficial wounds: re-epithelialization is always present, but however, if the vasculature is not disrupted at the moment of injury, the blood clot is not formed and angiogenesis may not be necessary. Coupling the closure model with angiogenesis is a topic of further research. It is likewise important to note that the principles of the present model could also be applied to actin purse string healing kinetics by just replacing the chemically induced closure rate by the mechanical stimulus sensed by cells [24] at the wound edge.

The simulations presented in this paper aim at providing new insights onto the kinetics of wound closure, in particular on the role of wound shape. Results agree with the observations made by Buck [10]: healing always starts at regions of high convexity. In very specific cases, an undesired retreat of the wound edge was observed at localized areas (the concave areas) of the wound. This effect may be avoided by a strict control of the closure rate parameters. However, it is worth noting that skin tension on head and neck wounds may induce not only changes on the wound shape but also an increase on the area [11]. Unfortunately, the results predicted here are rather qualitative, mainly due to the limited knowledge of the physiological 
parameters of the model. Until sufficient experimental work is done to capture the evolution of the wound edge, area, etc. for general wounds, the validation of the model will be difficult and the predictive power of the mathematical method will be limited.

Finally, regions of unsuccessful healing (i.e. incomplete) may be demarcated in function of the parameters. Using asymptotic solutions, we showed that healing is eventually aborted if the closure rate is not adequate. The thickness of the active layer and the wound shape play a critical role in this matter as well. A deeper characterization of the critical size defect may be accomplished by parametric studies of the present model.

Acknowledgments The authors gratefully acknowledge the Delft Centre for Materials, at the Delft University of Technology, for the financial support of this research.

Open Access This article is distributed under the terms of the Creative Commons Attribution Noncommercial License which permits any noncommercial use, distribution, and reproduction in any medium, provided the original author(s) and source are credited.

\section{Appendix: Data set of the numerical tests}

Of the physiological model parameters, only $D=5 \times 10^{-7} \mathrm{~cm}^{2} / \mathrm{s}[3]$ and $\lambda=$ $1.6 \times 10^{-5} 1 / \mathrm{s}[38]$ have been estimated. The authors are not aware of valid estimates for the other parameters, and hence these will be treated as fitting parameters in the calculations. In order to simplify the analysis, we fix $P=5 \times 10^{-7}[c] / \mathrm{s}$ (in which $[c]$ denotes the unit of the EGF concentration) and $\delta=0.1 \mathrm{~cm}$ throughout our calculations. The other parameters will be taken, depending on the analysis purposes, as summarized in Tables 2 and 3.

Table 2 Physiological parameters for the asymptotic solutions presented in Figs. 4 and 5

\begin{tabular}{llll}
\hline & Figure 4 & Figure 5a & Figure 5b \\
\hline $\begin{array}{l}\text { Geometry } \\
(\mathrm{cm})\end{array}$ & Circular & Circular & Circular \\
$r_{0}=0.5$ & $r_{0}=0.5$ & $r_{0}=0.5$ \\
$\theta([\mathrm{c}])$ & $2.5 \times 10^{-2}$ & $5 \times 10^{-4}$ & $\in\left[3 \times 10^{-5}, 7 \times 10^{-3}\right]$ \\
& $10^{-3}$ & \\
& & & $10^{-9}, 10^{-7}, 5 \times 10^{-7}$ \\
$\alpha(\mathrm{cm} / \mathrm{s})$ & $10^{-5}$ & & $10^{-6}, 2 \times 10^{-6}, 3 \times 10^{-6}$ \\
$\beta(\mathrm{cm} / \mathrm{s})$ & $2.1 \times 10^{-7}$ & $2.1 \times 10^{-7}$ & $4 \times 10^{-6}, 5 \times 10^{-6}, 7 \times 10^{-6}$ \\
$\Omega$ & {$[0,1.5]$} & {$[0,1.5]$} & 0 \\
\hline
\end{tabular}


Table 3 Physiological and numerical parameters used in the calculation of the results presented in Figs. 6, 7, 8 and 9

\begin{tabular}{|c|c|c|c|c|}
\hline & Figure 6 & Figure 7 & Figure 8 & Figure 9 \\
\hline $\begin{array}{l}\text { Geometry } \\
(\mathrm{cm})\end{array}$ & $\begin{array}{l}\text { Circular } \\
r_{0}=0.5\end{array}$ & $\begin{array}{l}\text { Circular } \\
r_{0}=0.5\end{array}$ & $\begin{array}{l}\text { Elliptical } \\
a=\sqrt{0.25 / A R} \\
b=a / A R \\
\mathrm{AR}=1, \ldots, 5\end{array}$ & $\begin{array}{l}\text { Starfish } \\
\begin{array}{l}a=0.25 \\
b=0.225\end{array}\end{array}$ \\
\hline$\theta([\mathrm{c}])$ & $8 \times 10^{-4}$ & $\in\left[0,10^{-3}\right]$ & $\in\left[0,3 \times 10^{-3}\right]$ & $\in\left[0,3 \times 10^{-3}\right]$ \\
\hline$\alpha(\mathrm{cm} / \mathrm{s})$ & $10^{-5}$ & $\in\left[10^{-5}, 10^{-4}\right]$ & $10^{-5}$ & 1 \\
\hline$\beta\left(\mathrm{cm}^{2} / \mathrm{s}\right)$ & $2.1 \times 10^{-7}$ & 0 & $\begin{array}{l}2.1 \times 10^{-7} \\
{\left[0, L_{x}\right] \times\left[0, L_{y}\right]}\end{array}$ & $0,0.05,0.1$ \\
\hline$\Omega$ & {$[0,1.5]$} & {$[0,1.5]$} & $\begin{array}{l}L_{x}=0.5+a \\
L_{y}=0.5+b \\
\text { AR, nx, ny } \\
1,24,24\end{array}$ & {$[-1,1] \times[-1,1]$} \\
\hline \# Internal & & & $2,29,20$ & nx, ny \\
\hline Nodes & 499 & 499 & $\begin{array}{l}3,33,19 \\
4,37,18 \\
5,39,17\end{array}$ & 50,50 \\
\hline refnratio & 3 & 3 & 3 & 3 \\
\hline$\varepsilon(\mathrm{cm})$ & $1.5 \times 10^{-3}$ & $1.5 \times 10^{-3}$ & $\approx 1.33 \times 10^{-2}$ & $1.31 \times 10^{-2}$ \\
\hline$\Delta t_{\max }(\mathrm{s})$ & 5 & 5 & 30 & 30 \\
\hline CFL & 0.15 & 0.15 & 0.15 & 0.45 \\
\hline
\end{tabular}

$A R$ the wound aspect ratio, $m x$ and $m y$ the number of internal nodes in the $x$ and $y$ directions, respectively

\section{References}

1. Adalsteinsson D, Sethian JA (1995) A fast level set method for propagating interfaces. J Comput Phys 118(2):269-277

2. Adam JA (1986) A simplified mathematical model of tumor growth. Math Biosci 81(2):229-244

3. Adam JA (1999) A simplified model of wound healing (with particular reference to the critical size defect). Math Comput Model 30(5-6):23-32

4. Adam JA (2002) The effect of surface curvature on wound healing in bone: II. the critical size defect. Math Comput Model 35(9-10):1085-1094

5. Arnold F, West DC (1991) Angiogenesis in wound healing. Pharmacol Theory 52(3):407-422

6. Arnold JS, Adam JA (1999) A simplified model of wound healing-II: the critical size defect in two dimensions. Math Comput Model 30(11-12):47-60

7. Britton NF (2003) Essential mathematical biology. Springer, London

8. Britton NF, Chaplain MA (1993) A qualitative analysis of some models of tissue growth. Math Biosci 113(1):77-89 
9. Brock J, Midwinter K, Lewis J, Martin P (1996) Healing of incisional wounds in the embryonic chick wing bud: characterization of the actin purse-string and demonstration of a requirement for rho activation. J Cell Biol 135(4):1097-1107

10. Buck RC (1979) Cell migration in repair of mouse corneal epithelium. Invest Ophthalmol Vis Sci 18(8):767-784

11. Bush JA, Ferguson MWJ, Mason T, McGrouther DA (2008) Skin tension or skin compression? small circular wounds are likely to shrink, not gape. J Plast Reconstr Aesthet Surg 61(5):529-534

12. Chaplain MA (2000) Mathematical modelling of angiogenesis. J Neurooncol 50(1-2):37-51

13. Clark RA (1989) Wound repair. Curr Opin Cell Biol 1(5):1000-1008

14. Clark RAF, Ghosh K, Tonnesen MG (2007) Tissue engineering for cutaneous wounds. J Invest Dermatol 127(5):1018-1029

15. Gaffney EA, Pugh K, Maini PK, Arnold F (2002) Investigating a simple model of cutaneous wound healing angiogenesis. J Math Biol 45(4):337-374

16. Grasso S, Hernndez JA, Chifflet S (2007) Roles of wound geometry, wound size, and extracellular matrix in the healing response of bovine corneal endothelial cells in culture. Am J Physiol Cell Physiol 293(4):C1327-C1337

17. Greenspan EM (1972) Chemotherapy of breast cancer. N Engl J Med 287(22):1150

18. Hogea CS, Murray BT, Sethian JA (2006) Simulating complex tumor dynamics from avascular to vascular growth using a general level-set method. J Math Biol 53(1):86-134

19. Javierre E, Vuik C, Vermolen FJ, Segal A (2007) A level set method for three dimensional vector stefan problems: dissolution of stoichiometric particles in multi-component alloys. J Comput Phys 224(1):222-240

20. Lu L, Reinach PS, Kao WW (2001) Corneal epithelial wound healing. Exp Biol Med (Maywood) 226(7):653-664

21. Malladi R, Sethian JA (1998) A real-time algorithm for medical shape recovery. In: Proc. Sixth International Conference on Computer Vision, 4-7 Jan, pp 304-310

22. Martin P, Lewis J (1992) Actin cables and epidermal movement in embryonic wound healing. Nature 360(6400):179-183

23. Mattila PK, Lappalainen P (2008) Filopodia: molecular architecture and cellular functions. Nat Rev Mol Cell Biol 9(6):446-454

24. Moreo P, García-Aznar JM, Doblaré M (2008) Modeling mechanosensing and its effect on the migration and proliferation of adherent cells. Acta Biomaterialia 4(3):613-621

25. Murray JD (2003) Mathematical biology: II Spatial models and biomedical applications, vol 18, 3rd edn. Springer, New York

26. Odland G, Ross R (1968) Human wound repair. I. Epidermal regeneration. J Cell Biol 39(1):135-151

27. Olsen L, Sherratt JA, Maini PK (1995) A mechanochemical model for adult dermal wound contraction and the permanence of the contracted tissue displacement profile. J Theor Biol 177(2): $113-128$

28. Osher S, Fedkiw R (2003) Level set methods and dynamic implicit surfaces, vol 153. Springer, New York

29. Osher S, Sethian JA (1988) Fronts propagating with curvature-dependent speed: algorithms based on hamilton-jacobi formulations. J Comput Phys 79(1):12-49

30. Peng D, Merriman B, Osher S, Zhao H, Kang M (1999) A pde-based fast local level set method. J Comput Phys 155(2):410-438

31. Rodriguez PG, Felix FN, Woodley DT, Shim EK (2008) The role of oxygen in wound healing: a review of the literature. Dermatol Surg, May

32. Ross R, Odland G (1968) Human wound repair. II. Inflammatory cells, epithelial-mesenchymal interrelations, and fibrogenesis. J Cell Biol 39(1):152-168

33. Sethian JA (1999a) Fast marching methods. SIAM Rev 41(2):199-235

34. Sethian JA (1999b) Level set methods and fast marching methods, vol 3, 2nd edn. Cambridge University Press, Cambridge

35. Sherratt JA, Dallon JC (2002) Theoretical models of wound healing: past successes and future challenges. C R Biol 325(5):557-564

36. Sherratt JA, Martin P, Murray JD, Lewis J (1992) Mathematical models of wound healing in embryonic and adult epidermis. IMA J Math Appl Med Biol 9(3):177-196

37. Sherratt JA, Murray JD (1991) Mathematical analysis of a basic model for epidermal wound healing. J Math Biol 29(5):389-404 
38. Sherratt JA, Murray JD (1992) Epidermal wound healing: the clinical implications of a simple mathematical model. Cell Transplant 1(5):365-371

39. Shymko RM, Glass L (1976) Cellular and geometric control of tissue growth and mitotic instability. J Theor Biol 63(2):355-374

40. Singer AJ, Clark RA (1999) Cutaneous wound healing. N Engl J Med 341(10):738-746

41. Stadelmann WK, Digenis AG, Tobin GR (1998) Physiology and healing dynamics of chronic cutaneous wounds. Am J Surg 176(2, Supplement1):26S-38S

42. Sussman M, Smereka P, Osher S (1994) A level set approach for computing solutions to incompressible two-phase flow. J Comput Phys 114(1):146-159

43. Van der Zwaag S (2007) Self healing materials: an alternative approach to 20 centuries of materials science. Springer Series in Materials Science, vol 100. Springer Netherlands, Dordrecht, ISBN 978-1-4020-6249-0

44. Vermolen FJ, van Baaren E, Adam JA (2006) A simplified model for growth factor induced healing of wounds. Math Comput Model 44(9-10):887-898

45. Wearing HJ, Sherratt JA (2000) Keratinocyte growth factor signalling: a mathematical model of dermal-epidermal interaction in epidermal wound healing. Math Biosci 165(1):41-62

46. Zhao $\mathrm{H}$ (2005) A fast sweeping method for eikonal equations. Math Comp 74(250):603-627 (electronic) 